Cahiers $d u$ MONDE RUSSE

\section{Cahiers du monde russe}

Russie - Empire russe - Union soviétique et États indépendants

$43 / 1 \mid 2002$

Varia

\title{
À propos de la création du collège des Affaires étrangères sous Pierre le Grand.
}

\author{
Anna Joukovskaïa-Lecerf
}

\section{OpenEdition}

\section{Journals}

Édition électronique

URL : https://journals.openedition.org/monderusse/8480

DOI : $10.4000 /$ monderusse. 8480

ISSN : $1777-5388$

Éditeur

Éditions de l'EHESS

Édition imprimée

Date de publication : 1 janvier 2002

Pagination : $57-66$

ISBN : 2-7132-1773-3

ISSN : $1252-6576$

Référence électronique

Anna Joukovskaïa-Lecerf, «À propos de la création du collège des Affaires étrangères sous Pierre le Grand. », Cahiers du monde russe [En ligne], 43/1 | 2002, mis en ligne le 01 janvier 2007, consulté le 03 septembre 2022. URL : http://journals.openedition.org/monderusse/8480 ; DOI : https://doi.org/ 10.4000/monderusse.8480 
chercher : repérer : avancer

Cet article est disponible en ligne à l'adresse :

http://www.cairn.info/article.php?ID REVUE=CMR\&ID NUMPUBLIE=CMR 431\&ID ARTICLE=CMR 4310057

\title{
À propos de la création du collège des Affaires étrangères sous Pierre le Grand
}

\author{
par Anna JOUKOVSKAÏA-LECERF
}

\section{Editions de l'EHESS | Cahiers du monde russe}

\author{
2002/1 - Vol 43 \\ ISSN 1252-6576 | ISBN 2713217733 | pages 57 à 66
}

\footnotetext{
Pour citer cet article :

—JOUKOVSKAÏA-LECERF A., À propos de la création du collège des Affaires étrangères sous Pierre le Grand, Cahiers du monde russe 2002/ 1, Vol 43, p. 57-66.
} conditions générales d'utilisation du site ou, le cas échéant, des conditions générales de la licence souscrite par votre établissement. Toute autre reproduction ou représentation, en tout ou partie, sous quelque forme et de quelque manière que ce soit, est interdite sauf accord préalable et écrit de l'éditeur, en dehors des cas prévus par la législation en vigueur en France. Il est précisé que son stockage dans une base de données est également interdit. 


\section{À PROPOS DE LA CRÉATION DU COLLÈGE DES AFFAIRES ÉTRANGÈRES SOUS PIERRE LE GRAND}

Le rôle du collège des Affaires étrangères dans la gestion de la politique extérieure et de la diplomatie de la Russie, durant toute l'existence de cette institution (1717 - 1832), fut paradoxalement imprécis : formellement, le collège était le principal rouage des relations internationales du pays, mais il fut presque constamment éclipsé, d'abord par le Conseil suprême secret, puis par le Cabinet de l'impératrice, et ensuite par l'Assemblée des ministres et généraux, par la Conférence auprès de la Cour impériale et par le Conseil d'État. De façon encore plus paradoxale, le chef de la diplomatie russe, le chancelier (ou, à certaines périodes, le vice-chancelier), fut toujours le premier à trahir les intérêts du collège, dont il restait pourtant président, en l'écartant des affaires importantes dès qu'il entrait luimême dans les structures politiques citées.

L'omniprésence de Pierre ${ }^{e r}$, habitué à diriger personnellement sa diplomatie, est sans doute la principale raison qui empêcha le collège d'acquérir une autorité incontestable dans les Affaires étrangères. Par ailleurs, des phénomènes propres à l'organisation interne du collège, telle qu'elle émergea pendant la réforme (1717 1720), ont sans doute freiné sensiblement le développement de la puissance potentielle de cette institution. Ils ont été moins étudiés et il conviendrait de les examiner de plus près.

Depuis 1709 , le service diplomatique russe fut géré par un chancelier (le comte Gavriil Golovkin) et un vice-chancelier (Petr Šafirov) ${ }^{1}$, directement subordonnés

1. N. A. Voskresenskij, ed., Zakonodatel'nye akty Petra I (Actes législatifs de Pierre Ier), Moscou - Leningrad, $1945, \mathrm{n}^{\circ} 404, \mathrm{p} .517$. 
au tsar. Ils avaient à leur service une chancellerie des Ambassades installée dès 1710 à Saint-Pétersbourg 2 .

Pierre Irr, depuis le début de la Guerre du Nord, prit l'habitude de correspondre directement avec ses ministres à l'étranger sur des questions importantes. Golovkin et Šafïrov, secondés par les commis de la chancellerie des Ambassades, vaquaient surtout aux affaires courantes et servaient de représentants du tsar devant les ministres étrangers. «Les premiers représentants diplomatiques russes - écrit Aleksandrenko - recevaient des instructions et des conseils de leur souverain, étaient responsables devant lui et ne craignaient que lui seul. L'influence des institutions centrales et même des personnes qui dirigeaient les Affaires étrangères (chancelier, vice-chancelier) était insignifiante. $»^{3}$

Pierre Ier n'avait pas de conseil permanent, mais il lui arrivait de réunir autour de lui des diplomates et des généraux pour tenir des consultations sur les affaires extérieures. À partir de 1710, ces conseils de campagne étaient désignés, dans les documents de la chancellerie des Ambassades, sous le nom de Conseil privé (Tajnyj sovet).

Rien ne prouve que cette organisation ne répondait plus aux besoins du moment ${ }^{5}$, lorsque, en 1717 , le tsar annonça la création d'un collège des Affaires étrangères ${ }^{6}$ qui devait remplacer la chancellerie des Ambassades et le secrétariat des Ambassades. Au fond, Pierre Ier, qui de toute façon tenait tous les fils de sa diplomatie dans ses propres mains, aurait pu se passer de la réforme du département extérieur. Mais ce monarque, qui appréciait l'uniformité dans l'architecture,

2. L'origine de la chancellerie remonte au début de la Guerre du Nord quand Pierre Ier, souvent absent de Moscou, fit détacher du secrétariat des Ambassades une chancellerie itinérante des Ambassades, petit bureau mobile qui pouvait le sujvre sur le théâtre de la guerre et maintenir sa correspondance diplomatique. Durant la guerre, elle exécutait les affaires les plus importantes et les plus urgentes, travaillant sous l'étroite supervision du tsar. Elle éclipsa progressivement le secrétariat des Ambassades, qui resta définitivement à Moscou et auquel on ne confiait plus que des tâches d'importance mineure. L'organisation de la chancellerie des Ambassades ne subit pas de changements notables jusqu'en 1720, date d'entrée en vigueur du Règlement général des collèges et des actes réglementaires concernant le collège des Affaires étrangères.

3. V. N. Aleksandrenko, Russkie diplomatičeskie agenty $v$ Londone $v$ XVIII veke (Agents diplomatiques russes à Londres au XVII' s.), Varsovie, 1897, vol. 1, p. 15.

4. Les auteurs de l'histoire officielle du ministère des Affaires étrangères amplifient outre mesure le rôle dı Conseil privé quand ils écrivent que la voix décisive dans la direction de la diplomatie lui appartenait (S. A. Belokukrov et al., Očerk istorii Ministerstva insotrannyh del, 1802 - 1902 (Abrégé de l'histoire du ministère des Affaires étrangères, 1802 - 1902), SaintPétersbourg, 1902, p.37). Rappelons qu'aucun acte officiel parmi ceux qui sont connus aujourd'hui n'atteste l'institution d'un Conseil privé.

5. Les succès de la diplomatie pétrinienne des années $1700-1710$ sont attestés par tous les historiens. Citons quelques ouvrages de référence récents : Rossijskaja diplomatija v portretah (La diplomatie russe à travers des images de diplomates), Moscou, 1992 ; Istorija vnešnej politiki Rossii, XVIII vek (Histoire de la politique extérieure de la Russie, XVIII siècle), Moscou, 1998 ; Rossijskaja diplomatija : Istorija i sovremennost '. Materialy naučno-praktičeskoj konferencii, posvjaščennoj 450-letiju sozdanija Posol'skogo prikaza (La diplomatie russe : histoire et modernité. Actes de la conférence scientifique à l'occasion du $450^{\circ}$ anniversaire de la création du secrétariat des Ambassades), Moscou, 2001.

6. N. A. Voskresenskij, op. cit., n² 265, p. 220. 
reporta ses préférences sur l'administration et exigea que la façade de son gouvernement fût régulière. Puisqu'il avait décidé de donner à l'ensemble de son administration centrale une organisation collégiale à la suédoise ${ }^{7}$, le service diplomatique devrait s'y conformer tout comme les autres branches de l'administration.

Pierre $\mathrm{I}^{\mathrm{er}}$ savait que la réorganisation totale des secrétariats moscovites, les prikazy, était une affaire de plusieurs années. En revanche, le tsar voulait que les principaux détenteurs du pouvoir exécutif de son État adoptent promptement le principe de la direction collégiale à la suédoise. Il espérait que cette mesure pourrait diminuer les risques de gestion partiale ou incompétente.

Les premiers oukases qui exprimaient cette volonté étaient moins le résultat de réflexions théoriques sur la nature du pouvoir exécutif que des considérations concrètes du moment. En 1714, Pierre Ier veut s'assurer que les sénateurs, qu'il laisse gouverner le pays durant ses absences de la capitale, prennent leurs décisions ensemble et en endossent les conséquences. Non content de les exhorter à la responsabilité, le tsar se donne des moyens de contrôle en introduisant la pratique des procès-verbaux et de la signature obligatoire ${ }^{8}$.

Le même scénario se répète deux ans plus tard, cette fois avec la participation du chancelier et de ses collègues. Au printemps de 1716, Pierre I ${ }^{\mathrm{er}}$, accompagné de Golovkin, Šafirov, Petr Tolstoj et Grigorij Dolgorukij, se rend à Dantzig pour rencontrer le roi de Pologne, Auguste II. La rencontre des deux souverains devait être précédée par des pourparlers entre leurs représentants au sujet des griefs du tsar contre le roi et de la médiation projetée de la Russie entre Auguste II et la confédération ${ }^{9}$. Les négociations, commencées le 24 mars, s'annonçaient difficiles, et il était très important que les ministres du tsar agissent d'un commun accord. Pour les accoutumer à l'unanimité dans l'action et assurer la responsabilité collective, Pierre Ier écrit, le 5 avril 1716, un oukase qui entérine la procédure de composition et de signature des minutes, des procès-verbaux et des documents originaux que le «collège des Ambassades » (c'est ainsi qu'on appelait désormais le Conseil privé) ${ }^{10}$ pouvait adresser aux ministres russes ou étrangers ${ }^{11}$.

La composition du collège des Ambassades n'étant pas précisée dans le texte de l'oukase, seul le contexte historique de son apparition permet d'affirmer qu'il visait les quatre personnes déjà citées : le chancelier, le vice-chancelier et les conseillers privés actuels Dolgorukij et Tolstoj. Des sources d'archives de la fin de 1719 (début 1720) attestent que « suite à un oukase de S.M.I. » Dolgorukij et Tolstoj siégeaient «de temps en temps» avec le chancelier et le vice-chancelier, assistaient aux

7. Claes Peterson, Peter the Great's administrative and judicial reforms: Swedish antecedents and the process of reception, Stockholm, 1979, p. 67 et passim.

8. Oukase du 4 avril 1714 (N. A. Voskresenskij, op. cit., $n^{\circ} 250$, p. 209).

9. S. M. Solov'ev, Istorija Rossii s drevneǰ̌h vremen (Histoire de la Russie depuis I'Antiquité), Moscou, 1963, vol. 8, p. 433.

10. Le mot collège désigne ici une assemblée consultative ; à partir de 1717 ce mot définira des institutions administratives centrales dirigées chacune par un conseil

11. N. A. Voskresenskij, op. cit., $\mathrm{n}^{\circ} 405$, p. 517-518. 
conférences avec des ministres étrangers, examinaient les dépêches et signaient les résolutions du collège des Ambassades ${ }^{12}$.

Dolgorukij étant absent la plupart du temps, puisqu'il exerçait entre 1715 et 1721 les fonctions d'ambassadeur en Pologne, Golovkin, Šafirov et Tolstoj formaient un triumvirat qui dirigeait les affaires diplomatiques courantes. Leur cohabitation ne pouvait pas être paisible. L'incompatibilité de caractère entre Golovkin et Šafirov, leur appartenance à des réseaux politiques opposés (les Naryškin et les Saltykov) ${ }^{13}$, la concurrence qui existait entre eux, les amenaient à entretenir au sein de la chancellerie des Ambassades des groupes antagonistes de clients. Golovkin, qui faisait partie du cercle intime de Pierre I Ier depuis 1'enfance du tsar, occupait une position prépondérante par rapport à Šafirov. Vraisemblablement, ce dernier profitait parfois du soutien de Tolstoj, chef des Saltykov. Mais les liens qui les unissaient étaient corrodés par des rivalités de petits intérêts personnels ${ }^{14}$. Tel était l'équilibre fragile du département diplomatique quand la réforme collégiale entra dans sa phase décisive.

Le 15 décembre 1717, Pierre I ${ }^{\text {er }}$ nomma le chancelier Golovkin et le vice-chancelier Šafirov aux postes de président et de vice-président du collège des Affaires étrangères ${ }^{15}$. Celui-ci succéda au collège des Ambassades et à la chancellerie des Ambassades, tandis que le secrétariat des Ambassades fut relégué au rang de filiale, sous le nom de Bureau de Moscou. Quelques jours auparavant, le 11 décembre 1717 , le tsar avait déjà donné l'ordre aux présidents de procéder au recrutement du personnel et à l'organisation de leurs collèges, prenant pour exemple les collèges suédois ${ }^{16}$.

La structure des collèges suédois comportait deux niveaux. Un conseil des membres décisionnaires commandait une chancellerie constituée par des commis de différents rangs. Les conseils des collèges russes devaient être composés de dix membres: président, vice-président, quatre conseillers ${ }^{17}$, quatre assesseurs ${ }^{18}$. Malgré l'existence d'une hiérarchie fonctionnelle, l'obligation de participer à la prise de décision concernait tous les membres. Ils bénéficiaient chacun d'une voix (on allait adopter le système de vote majoritaire) ${ }^{19}$. Les présidents des collèges devraient choisir leurs futurs collaborateurs et les présenter au Sénat où s'effectuerait le choix définitif ${ }^{20}$.

\section{Ibid., $\mathrm{n}^{\circ} 409$, p. 530.}

13. John P. LeDonne, «Ruling families in the Russian political order », Cahiers du Monde russe et soviétique, XXVIII, 3-4, juillet-décembre 1987, p. 246, 249 et passim.

14. Cf. les dépêches du ministre du roi de France à Saint-Pétersbourg, Campredon, Sbornik imperatorskogo russkogo istoričeskogo obščestva, 40, p. $169 ; 41$, p. 11.

15. N. A. Voskresenskij, op. cit., $\mathrm{n}^{\circ} 265$, p. 220.

16. Ibid., $\mathrm{n}^{\circ} 261$, p. 217.

17. Au collège des Affaires étrangères ils allaient s'appeler conseillers de la chancellerie.

18. N. A. Voskresenskij, op. cit., $n^{\circ} 261$, p. 216.

19. Règlement général, article VI (ibid., $\mathrm{n}^{\circ} 401$, p. 487).

20. Ibid. 
Pour le président Golovkin, l'enjeu était de taille. Auparavant, un Tolstoj ou un Dolgorukij se mêlaient certes plus ou moins régulièrement de ses affaires. Mais il régnait du moins en maître à la chancellerie des Ambassades, où ses créatures étaient plus nombreuses que les fidèles de Šafirov. Désormais, on comptait l'entourer de huit nouveaux collègues permanents, tout en lui conférant un statut équivoque de primus inter pares. Occupées par des personnages importants, ces huit vacances deviendraient autant de fonctions traduisant l'influence personnelle en puissance administrative, sinon politique. Golovkin savait que même s'il parvenait, au début, à faire occuper ces places par des membres de son clan, son avenir ne serait pas assuré, parce que les changements de conjoncture dans le gouvernement russe étaient rapides et imprévisibles. Il serait plus prudent pour lui d'écarter les grands de son conseil, de le restreindre autant que possible et de le peupler d'obscurs commis anodins, dévoués et professionnellement efficaces.

D'après l'oukase du 11 décembre 1717, les collèges devaient entrer en fonction dès le début de 1719 , mais comme à la fin de 1718 rien n'était encore prêt, le tsar prolongea la période préparatoire d'un an.

Au début de 1719 , le chancelier proposa au Sénat les noms d'au moins deux personnes qu'il souhaitait voir membres de son conseil (les anciens sous-secrétaires Vasilij Stepanov et Petr Kurbatov). Le 19 mai de cette même année, il essaya d'appliquer les nouvelles procédures de vote majoritaire et de signature commune des procès-verbaux, mais il se heurta à un violent refus du vice-chancelier. Šafirov déclara que, tant que le conseil ne serait pas au complet, les nouveaux membres ne pourraient pas accéder à leurs fonctions, et quitta le conseil sans rien signer. Golovkin défendit au personnel du collège d'aller voir Šafirov ou d'exécuter ses ordres, et se dépêcha de communiquer le procès-verbal de la séance à Pierre Ir21 .

Le chancelier ne parvint pas à ternir la réputation de Šafirov dont les talents professionnels incontestables étaient connus et appréciés du tsar. En juin 1719, moins d'un mois après le scandale au collège, Pierre Ier décora Šafirov d'une étoile de l'ordre de Saint-André. En revanche, Golovkin contrôlait enfin entièrement la situation à l'intérieur du collège et se pressa d'en profiter.

Le 28 avril 1718, Pierre I $^{\text {er }}$ avait ordonné aux présidents de faire rédiger les règlements de leurs collèges ${ }^{22}$. À la fin de 1719 , il ne restait plus de temps à perdre, et Golovkin confia ce travail à Vasilij Stepanov, déjà cité. La tâche se révéla délicate : on devait se conformer aux articles du Règlement général (qui n'était pas encore promulgué, mais dont le texte était prêt et attendait la signature du tsar) et, d'un autre côté, il fallait protéger les intérêts des personnes en place et, avant tout, les prérogatives du président-chancelier. Nous venons de voir quelles pouvaient être les directives de Golovkin à Stepanov: écarter Tolstoj, Dolgorukij et les

21. Pour les détails du scandale racontés par les deux parties opposées, cf. S. M. Solov'ev, op. cit., 8, p. 456-457 ; S. A. Fejgina, Alandskij kongress. Vnešnjaja politika Rossii v konce Severnoj vojny (Le congrès d'Aland. La politique extérieure de la Russie à la fin de la Guerre du Nord), Moscou, 1959, p. 413-414.

22. N. A. Voskresenskij, op. cit., $n^{\circ} 49$, p. 60 . 
hommes de leur envergure et argumenter la nécessité de restreindre le nombre des membres du conseil.

Le brouillon des Statuts du collège des Affaires étrangères, rédigé par Stepanov, fut remanié par lui-même et par Golovkin au moins quatre fois avant d'être présenté au tsar. La comparaison des versions successives du passage qui nous intéresse (sur Tolstoj et Dolgorukij) met en évidence l'intention profonde des auteurs : éluder la discussion concernant ces personnages et l'éventuelle possibilité de leur donner des successeurs ${ }^{23}$.

Golovkin y parvint. Quand, le 13 février 1720, Pierre Irr ratifia le document intitulé De l'institution du collège des Affaires étrangères, on pouvait y lire ceci :

«Quand les affaires sont graves, inviter tous ou quelques-uns, selon l'importance de l'affaire, des conseillers privés actuels, et que chacun donne son conseil par écrit, et qu'on fasse le rapport de la décision ensuite. » ${ }^{24}$

La loi ne précisait pas quelles affaires devaient être considérées comme « graves », ni comment il fallait choisir « quelques-uns» des conseillers privés actuels parmi «tous ». Au lieu d'obtenir des places permanentes dans le conseil du collège des Affaires étrangères, les conseillers privés actuels devaient se contenter d'invitations épisodiques du monarque, comme par le passé, ou encore d'invitations du chancelier qui n'allait certes pas les déranger souvent.

Quant aux vacances des conseillers de la chancellerie et des assesseurs, elles étaient trop modestes pour attirer des personnages déjà influents et donc dangereux. En outre, ces postes étaient trop éloignés hiérarchiquement du chancelier pour qu'il puisse en redouter la concurrence. En revanche, Šafirov s'opposait à l'entrée au conseil des conseillers de la chancellerie et des assesseurs. Il avait déjà déclaré auparavant que le chancelier cherchait à placer au conseil ses créatures et qu'en général il était déshonorant de siéger avec d'anciens commis ${ }^{25}$. Il aurait préféré voir leur nombre réduit et leurs droits limités au minimum.

Le paragraphe sur les droits des conseillers de la chancellerie et des assesseurs connut de multiples rédactions. Stepanov, dont les intérêts étaient cette fois directement concernés, attachait une attention particulière à la formule de la future loi ${ }^{26}$. Au final, le nombre des conseillers de la chancellerie fut réduit de quatre à deux, et les assesseurs perdirent le droit de siéger au conseil quand on y traitait des affaires politiques et des affaires touchant aux relations extérieures. La seule raison officielle pour expliquer cette décision fut la nécessité de garder les affaires politiques et diplomatiques dans le plus grand secret.

23. Rossijskij Gosudarstvennyj Arhiv Drevnih Aktov à Moscou, cité infra RGADA, f. 370, op. 1, d. 12,1.4-5 ; N. A. Voskresenskij, op. cit, n ${ }^{\circ} 409$, p. 530-531 et n 406, p. 519.

24. N. A. Voskresenskij, op. cit., n 406, p. 520 (en russe dans l'original).

25. Ibid.

26. RGADA, f. 370 , op. 1, d. 12, 1. 4-6 ; N. A. Voskresenskij, op. cit., $\mathrm{n}^{\circ} 409$, p. 531, $\mathrm{n}^{\circ} 406$, p. $519-520, \mathrm{n}^{\circ} 407$, p. 523 . 
Après tant d'efforts réformateurs, quand le collège se rassembla pour tenir la première séance conforme aux nouvelles réglementations (le 16 février 1720), Golovkin se retrouva en compagnie de son vieux client, le conseiller de la chancellerie Stepanov, et de deux ennemis : le vice-chancelier Šafïrov et le conseiller privé de la chancellerie Andrej Osterman (1'ami intime de Šafirov, l'homme des Saltykov et l'une des principales figures de l'ambassade russe au congrès d'Aland dont les services furent appréciés par le tsar). C'était un match nul.

Toutefois, d'après le Règlement général, la voix présidentielle était prépondérante $^{27}$. En outre, la position de Šafirov à la Cour se dégradait parce qu'il avait au moins autant d'ennemis que d'amis, et Osterman venait tout juste d'obtenir ce poste, son premier poste important dans le gouvernement central. Compte tenu des circonstances, Golovkin pouvait considérer que le résultat quelque peu déconcertant de ses efforts n'était, finalement, pas tant en sa défaveur. Quant au principe, tant redouté, de la direction collégiale, il ne pouvait certainement pas faire beaucoup de progrès dans ces conditions.

Le chancelier et le vice-chancelier s'opposaient à la constitution d'un conseil influent au sein de leur collège parce qu'ils ambitionnaient un autre projet, celui de l'instauration auprès du monarque d'un Conseil privé pour les Affaires étrangères.

Des projets de ce genre furent discutés dans des cercles proches du tsar tout au long de la réforme ${ }^{28}$. Pierre Ier lui-même y participa en 1715 et en 1717 , quand il émit l'idée de la création d'une institution semblable au kanslikollegium suédois : collège de la chancellerie des Affaires étrangères, ou «Collège politique où sont préparés tous les oukases, les lettres patentes et les ordres de mission (narjady ljudem) $»^{29}$.

Le chancelier et ses collègues ont manifesté à plusieurs reprises leur approbation de l'idée du Conseil privé. Dans le projet du règlement du collège des Affaires étrangères de la fin de 1719, Stepanov se trompe (et Golovkin ne le corrige pas) en écrivant que les conseillers de la chancellerie demanderont les résolutions sur les affaires « aux ministres civils du Conseil privé » ${ }^{30}$, tandis que, logiquement, il aurait dû écrire : «aux membres du conseil du collège des Affaires étrangères » ${ }^{31}$. Quelques mois plus tard, Osterman qui composait, sur la demande de Pierre ${ }^{\mathrm{er}}$, un projet de la Table des rangs ${ }^{32}$, insistait longuement sur l'idée que les États dont les

27. Règlement général, article VI (N. A. Voskresenskij, op. cit., $\mathrm{n}^{\circ} 401, \mathrm{p} .487$ ).

28. P. N. Miljukov, Gosudarstvennoe hozjajstvo Rossii v pervoj četverti XVIII stoletija i reforma Petra Velikogo ( $L$ 'administration de l'économie et des finances de la Russie au premier quart du XVIII siècle et la réforme de Pierre le Grand), Saint-Pétersbourg, 1905, p. 675-676.

29. N. A. Voskresenskij, op. cit, $\mathrm{n}^{\circ} 263$, p. 218 (en russe dans l'original); Claes Peterson, op. cit., p. $62,64,392$.

30. N. A. Voskresenskij, op. cit., $\mathrm{n}^{\circ} 409$, p. 531 (en russe dans l'original).

31. Cf. le même quiproquo dans le projet des Statuts du collège de 1722 (RGADA, f. 370, op. 1, d. $12,1.113 \mathrm{ob}$.).

32. S. M. Troickij, Russkij absoljutizm i dvorjanstvo $v$ XVIII veke : formirovanie bjurokatii ( $L$ 'absolutisme russe et la noblesse au XVIII siècle : la formation d'une bureaucratie), Moscou, 1974, p. 50-52. 
monarques se faisaient assister par un conseil étaient beaucoup mieux gouvernés que les autres, il donnait des exemples et citait même le livre des Proverbes ${ }^{33}$.

Ces insinuations ne furent pas entendues. En 1722, avec la réorganisation du Sénat et la promulgation de la Table des rangs, la réforme paraissait toucher à sa fin, et l'espoir de l'institution d'un Conseil privé devenait de plus en plus faible. Le tsar décidait des affaires extérieures comme auparavant, soit tout seul, soit en consultation avec ses conseillers privés qu'il invitait (jamais de façon régulière) dans son Cabinet ou au collège des Affaires étrangères.

Dans cette situation les membres du collège des Affaires étrangères jugèrent qu'il serait bon de garantir le prestige et le potentiel politique de leur conseil contre une éventuelle déflation politique. À cette époque où la société russe apprenait à mesurer l'influence et le pouvoir des serviteurs civils et militaires par leurs rangs dans la Table, cela aurait pu se faire assez naturellement. Les membres du conseil avaient alors tous de très hauts rangs : Golovkin le $1^{\text {er }}$ (en tant que chancelier; les présidents ayant seulement droit au $4^{\mathrm{e}} \mathrm{rang}$ ), Šafïrov le $2^{\mathrm{e}}$ (grâce à sa nomination, en 1722 , au grade de conseiller privé actuel ; le titre de vice-chancelier ne fut pas introduit dans la Table et les vice-présidents furent placés au $5^{\mathrm{e}}$ rang), Osterman le $4^{\mathrm{e}}$ (conseiller privé depuis 1721, tandis qu'il était entré au conseil au grade de conseiller privé de la chancellerie qui donnait droit seulement au $6^{\mathrm{e}}$ rang), Stepanov le $6^{\mathrm{e}}$ (conseiller privé de la chancellerie depuis 1721). Il aurait suffi de fixer par la loi cette configuration avantageuse.

Dans un projet des Statuts du collège rédigé en $1722^{34}$, on tenta de bloquer l'accès du conseil du collège des Affaires étrangères aux fonctionnaires de rang inférieur. On y demandait en outre d'élever le nombre de membres permanents de quatre à cinq personnes. Ce projet ne fut jamais réalisé.

En 1723, la Cour suprême condamna le vice-chancelier, accusé de graves manquements au règlement du Sénat, à la peine de mort que le tsar commua en exil perpétuel. Golovkin se sentit enfin soulagé, mais Šafirov fut rapidement remplacé par Osterman, nommé au poste de vice-chancelier sans en porter le titre. Intelligent, expérimenté, prudent, rusé et travailleur, il pouvait être plus dangereux pour le vieux chancelier que ne l'était Šafirov. Toutefois, l'idée de faire élever le conseil de leur collège au statut de Conseil privé les réunissait, pour le moment.

En 1724, Osterman présenta au tsar un projet de réforme du collège qui commençait par une affirmation catégorique :

« Les affaires du ressort du collège des Affaires étrangères, ou, pour dire simplement, du conseil privé, sont très importantes [...]. » ${ }^{35}$

Ce projet ne fut jamais approuvé ${ }^{36}$ : en 1725 , Pierre le Grand mourut et un autre règne commença, celui de Catherine Ire et du Conseil suprême secret, dont

33. RGADA, f. 370, op. 1, d. 16,1.50-52 ob.

34. Ibid., d. 12,1.110-131 ob. Date hypothétique.

35. S. A. Belokurov et al., op. cit., p. 44. En russe dans l'original.

36. Arhiv Vnešnej Politiki Rossijskoj Imperii - AVPRI, f. 2, op. 6, d. 7,1. 1 ob. 
Golovkin et Osterman devinrent membres en même temps que cinq autres hommes d'État éminents.

Le collège des Affaires étrangères céda une partie de ses pouvoirs au Conseil suprême secret, ses meilleurs cadres furent transférés à la chancellerie de ce dernier, et le collège se transforma, pour de longues années à venir, en un simple bureau.

La possibilité d'élever le conseil du collège des Affaires étrangères au rang d'un véritable lieu politique avait pourtant été réelle. Tel qu'il avait été défini, le conseil collégial aurait pu acquérir et maintenir une influence politique durable : il aurait été composé de la première autorité civile de l'État, de son adjoint, de deux ou trois dignitaires importants, épaulés par cinq ou six professionnels de l'administration diplomatique, et, en outre, les lois de l'époque lui auraient garanti une grande autonomie par rapport au Sénat ${ }^{37}$.

Mais Golovkin et Šafirov s'étaient opposés, pendant la création du collège des Affaires étrangères, à l'application stricte des principes fondateurs de la réforme. Ils étaient trop habitués à considérer la chancellerie des Ambassades comme leur fief et, en outre, ils espéraient devenir membres d'un conseil présidé par le tsar. Le conseil collégial qu'ils avaient composé était trop restreint pour être représentatif et l'influence politique personnelle de ses membres trop faible ${ }^{38}$ pour placer le collège au sommet du système de gestion des Affaires étrangères au moment où la mort de Pierre Ier y créa un vide.

Centre d'études du monde russe, soviétique et post-soviétique

54, boulevard Raspail

75006 Paris

lecerf@gage.polytechnique.fr

37. V. N. Aleksandrenko, op. cit., vol. 1, p. 485 ; S. M. Solov'ev, op. cit., vol. 9, p. 572 ; N. A. Voskresenskij, op. cit., n 69, p. 71.

38. Ainsi par exemple, le prince Boris Kurakin, conseiller privé et ambassadeur, avait plus d'autorité sur le corps diplomatique russe que le chancelier ; encouragé par le tsar, il dirigea, de son poste à La Haye, l'activité de ses collègues aussi bien avant qu'après l'institution du collège (Dan Altbauer, The diplomats of Peter the Great, 1689-1725, Harvard University, 1976 (thèse inédite), p. 83, 144 et passim). 